\title{
Hemorragia masiva: una complicación tardía del reemplazo de la gastrostomía endoscópica percutánea. A propósito de un caso
}

\author{
Massive hemorrhage: a late complication of replacement percutaneous \\ endoscopic gastrostomy: case report
}

\author{
Prof. asist. Dr. Tamer Sekmenlia, Prof. asist. Dr. Metin Gündüza, Dr. Hikmet Akbulut ${ }^{b}$, \\ Prof. adj. Dr. H. Haldun Emiroğluc, Prof. adj. Dr. Mustafa Koplay y Prof. adj. Dr. Ilhan Ciftci. ${ }^{a}$
}

\section{RESUMEN}

La gastrostomía endoscópica percutánea (GEP) se utiliza como alternativa de la alimentación enteral/nasoenteral en situaciones en las que la alimentación por vía oral a largo plazo no es eficaz o no se tolera. Se prefiere principalmente en pacientes con afecciones neurológicas y, además, como apoyo de la nutrición en pacientes con enfermedades cardíacas congénitas, fibrosis quística, enfermedad intestinal inflamatoria y diversas enfermedades orofaríngeas. Si bien la colocación es sencilla en comparación con muchos procedimientos invasivos, presenta complicaciones, que incluyen desde la infección de la herida hasta la muerte.

La GEP exige personal médico experimentado, antibióticos profilácticos adecuados e información exhaustiva para los pacientes o sus familias sobre el procedimiento y los cuidados posteriores. Presentamos una complicación rara, aunque importante, que surgió durante el reemplazo de la sonda de gastrostomía después del método de "corte y empuje". El tope, que debellegar hasta el extremo distal del estómago, se desplazó hacia arriba, hasta el esófago proximal, y causó una úlcera profunda en la mucosa esofágica y una hemorragia masiva. Palabras clave: Gastrostomía endoscópica percutánea, hemorragia digestiva, técnica de corte y empuje.

http: / / dx.doi.org/10.5546/ aap.2018.e315

Texto completo en inglés:

http:/ / dx.doi.org/10.5546/ aap.2018.eng.e315

Cómo citar: Sekmenli T, Gündüz M, Akbulut H, et al. Hemorragia masiva: una complicación tardía del reemplazo de la gastrostomía endoscópica percutánea. A propósito de un caso. Arch Argent Pediatr 2018;116(2):e315-e318.

a. Facultad de Medicina de Selçuk Üniversitesi, Departamento de Cirugía Pediátrica. Turquía.

b. Facultad de Medicina de Selçuk Üniversitesi, Departamento de Pediatría. Turquía.

c. Facultad de Medicina de Selçuk Üniversitesi, Departamento de Gastroenterología Pediátrica. Turquía.

d. Facultad de Medicina de Selçuk Unniversitesi, Departamento de Radiología. Turquía.

Correspondencia:

Dr. Tamer Sekmenli: dr_sekmenli@hotmail.com

Financiamiento: Ninguno.

Conflicto de intereses: Ninguno que declarar.

Recibido: 1-7-2017

Aceptado: 19-9-2017

\section{INTRODUCCIÓN}

Los pacientes con una nutrición deficiente o reducida son más propensos a sufrir trastornos del sistema inmunitario, dificultad para cicatrizar heridas y desequilibrio psicológico. ${ }^{1} \mathrm{El}$ inicio de la alimentación enteral a su debido tiempo plantea ventajas, como reducir la atrofia de la mucosa, proteger la función de la barrera intestinal y disminuir la traslocación bacteriana intestinal. ${ }^{2}$ Por lo tanto, cuando la alimentación por vía oral no es suficiente durante períodos prolongados (de 4 a 8 semanas), la enterostomía es de suma importancia para el apoyo nutricional de los pacientes con un trastorno funcional del aparato digestivo. Entre las clases de enterostomía, la gastrostomía es la más adecuada en función de la fisiología digestiva y puede introducirse mediante endoscopía percutánea o cirugía, o con métodos de radiología intervencionista. ${ }^{3}$

La gastrostomía endoscópica percutánea (GEP), descrita por primera vez por Gauderer y col. en la década del ochenta, es un método que se utiliza cuando el aparato digestivo está intacto, pero la alimentación por vía oral no es posible durante un período prolongado. ${ }^{4} \mathrm{Si}$ bien se utiliza con frecuencia en niños con secuelas neurológicas, también se prefiere en el caso de pacientes con enfermedades metabólicas y oncológicas, y trastornos anatómicos y funcionales de la parte superior del aparato digestivo. ${ }^{5} \mathrm{Al}$ igual que en cualquier procedimiento invasivo, pueden surgir complicaciones durante el procedimiento de GEP y después. Estas incluyen infección y pérdida periestomal, peritonitis, neumoperitoneo, hemorragia, obstrucción de la sonda y fistulización. La GEP, que el personal médico experimentado puede realizar con facilidad, ha evitado la práctica de la gastrostomía quirúrgica. ${ }^{6} \mathrm{Si}$ bien suele preferirse en pacientes con problemas neurológicos, puede utilizarse como apoyo de la nutrición en pacientes con enfermedades cardíacas congénitas, fibrosis 
quística, enfermedad intestinal inflamatoria y diversas enfermedades orofaríngeas. La GEP es una técnica viable y mínimamente invasiva; $\sin$ embargo, existen algunos riesgos inherentes de complicaciones leves y graves, que incluyen desde la infección de la herida hasta la muerte. ${ }^{7} \mathrm{~A}$ fin de evitar estas complicaciones, la GEP exige personal médico experimentado, antibióticos profilácticos adecuados e información exhaustiva para los pacientes o su familia sobre el procedimiento y los cuidados posteriores.

Presentamos una complicación rara, aunque importante, que se produjo durante el reemplazo de la sonda de gastrostomía después del método de "corte y empuje" . ${ }^{8-10} \mathrm{El}$ tope, que debe llegar hasta el extremo distal del estómago, se desplazó hacia arriba, hasta el esófago proximal, penetró en la mucosa esofágica y causó una úlcera profunda y una hemorragia masiva posterior.

\section{CASO}

Un niño de tres años y medio con una gastrostomía ingresó a la unidad de cuidados intensivos (UCI) con insuficiencia respiratoria por aspiración pulmonar durante la administración de los medicamentos, la que le originó un paro respiratorio. Era uno de los trillizos que nacieron por cesárea a las 24 semanas de edad gestacional, con 500 gramos de peso al nacer. Se le estaba realizando un seguimiento por el diagnóstico de parálisis cerebral, epilepsia, reflujo gastroesofágico y displasia broncopulmonar. La primera GEP se realizó cuando el niño tenía 8 meses. Dos meses antes de la internación actual, la sonda de la GEP se reemplazó mediante la técnica de "corte y empuje", y se le estaba administrando alimentación por dicha sonda sin ningún inconveniente.

$\mathrm{Al}$ momento del ingreso a la UCI, el estado general del paciente era bueno, con los siguientes resultados del examen físico: temperatura corporal de $36,5^{\circ} \mathrm{C}$, frecuencia respiratoria de 20 / min, frecuencia cardíaca de $110 / \mathrm{min}$, peso de $10 \mathrm{~kg}$ (percentil 25), altura de $84 \mathrm{~cm}$ (percentil 25) y perímetro cefálico de $41,5 \mathrm{~cm}$ (percentil 25). En la auscultación del tórax, se detectaron crepitantes gruesos. Se le había colocado una sonda de GEP en el lado izquierdo del epigastrio, y no se observaron complicaciones alrededor del estoma. El abdomen estaba blando y la palpación no le producía dolor. El examen neurológico del paciente reveló mayor tono muscular en los miembros superiores e inferiores, junto con signo de Babinski positivo y clonus. En cuanto el paciente se estabilizó, se inició el tratamiento con ampicilina y sulbactam, y la administración de líquido por vía intravenosa con un diagnóstico de neumonía por aspiración.

Cuando el estado general del paciente mejoró y los síntomas respiratorios disminuyeron, se comenzó la alimentación enteral a través de la sonda de GEP; sin embargo, la alimentación se interrumpió después de un vómito explosivo con sangre. Se introdujo una sonda nasogástrica, se administró solución salina para irrigación a través de la sonda de gastrostomía y se inició el tratamiento con ranitidina. Posteriormente, los drenajes de la sonda nasogástrica y de GEP fueron transparentes. Debido a que el valor de hemoglobina de control era de $7 \mathrm{~g} /$ dl, se transfundió una suspensión de eritrocitos $(10 \mathrm{ml} / \mathrm{kg})$. Se introdujo un catéter venoso central con guía de ecografía a través de la vena subclavia derecha y, durante el control radiográfico de la colocación del catéter, se observó una lesión similar a un bulto a la altura del esófago proximal. La hemorragia se atribuyó a este bulto. La tomografía computarizada de tórax que se realizó después reveló un cuerpo extraño incrustado, ubicado en el tercio proximal del esófago, sin ninguna fistulización con los vasos principales. Posteriormente, se realizó una endoscopía digestiva alta; el cuerpo

FIGURA 1. Cuerpo extraño en la esofagografía con contraste

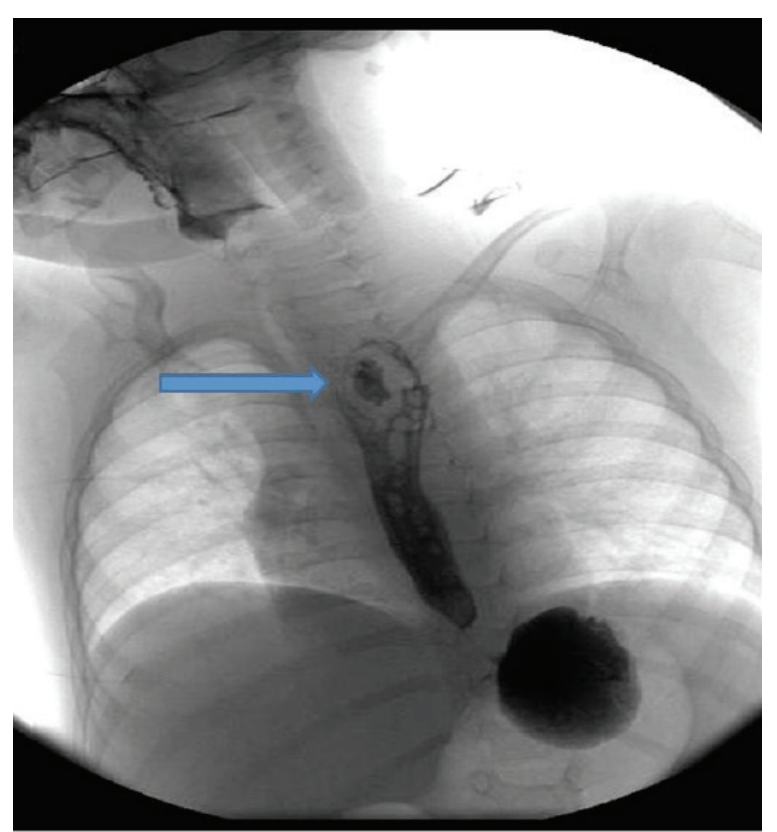


extraño estaba compuesto por partes del tope de la sonda de GEP anterior. La extracción endoscópica del cuerpo extraño se logró sin complicaciones (Figuras 1 y 2 a y $b$ ). Después del procedimiento, se controló la integridad del esófago con un estudio de radiografías del tránsito esofagogastroduodenal con contraste soluble en agua, y no se observaron pérdidas. Sin embargo, el paciente tuvo tres episodios más de hemorragia moderada intermitente, que se estabilizaron con apoyo médico conservador. Finalmente, el paciente murió después de un episodio de hemorragia abundante.

\section{DISCUSIÓN}

Para una vida saludable, es necesario comenzar una dieta balanceada y adecuada desde el nacimiento. La nutrición inadecuada ocasiona diversas enfermedades y complicaciones relacionadas. En los niños especialmente, además del retraso en el crecimiento, también se perjudica el desarrollo neuromotor. En consecuencia, para evitar la desnutrición, si la alimentación por vía oral es insuficiente o inadecuada, se utilizan métodos enterales / parenterales. Debido a que es el método más fácil de colocar, además de ser fisiológico y de conservar la mucosa y la flora intestinal, la alimentación enteral es la forma preferida de nutrición. ${ }^{6}$

Pueden emplearse métodos nasogástricos, nasoduodenales o nasoyeyunales. Sin embargo, para los pacientes que necesitan nutrición enteral a largo plazo, estos métodos pueden presentar muchas desventajas, por ejemplo, trastornos nasofaríngeos y erosiones del tejido, infecciones respiratorias de vías altas, esofagitis, erosión y úlceras gástricas, y ruptura de várices esofágicas. ${ }^{11}$ Por lo tanto, se considera la gastrostomía como una opción cuando el paciente no puede alimentarse por vía oral durante períodos prolongados, de más de 4 a 8 semanas. En la actualidad, los gastroenterólogos y cirujanos pediátricos realizan de forma generalizada la GEP por ser un procedimiento fácil de realizar, seguro y mínimamente invasivo., ${ }^{412}$ Cuando la realiza personal médico experimentado, la GEP tiene bajas tasas de complicaciones y es un procedimiento conveniente tanto para los pacientes como para los cuidadores. Si bien la GEP se lleva a cabo con mayor frecuencia en pacientes con trastornos neurológicos, también se utiliza para pacientes con enfermedades metabólicas, enfermedades cardíacas congénitas, fibrosis quística, enfermedades oncológicas y diversas enfermedades orofaríngeas, en las que la alimentación por vía oral es inadecuada. ${ }^{13}$

Aunque la GEP tiene una amplia aceptación por ser un método simple y confiable, pueden surgir complicaciones durante el procedimiento y después. ${ }^{6}$ Estas complicaciones se clasifican como graves y leves. Las complicaciones graves de la GEP incluyen perforación gástrica, hemorragia

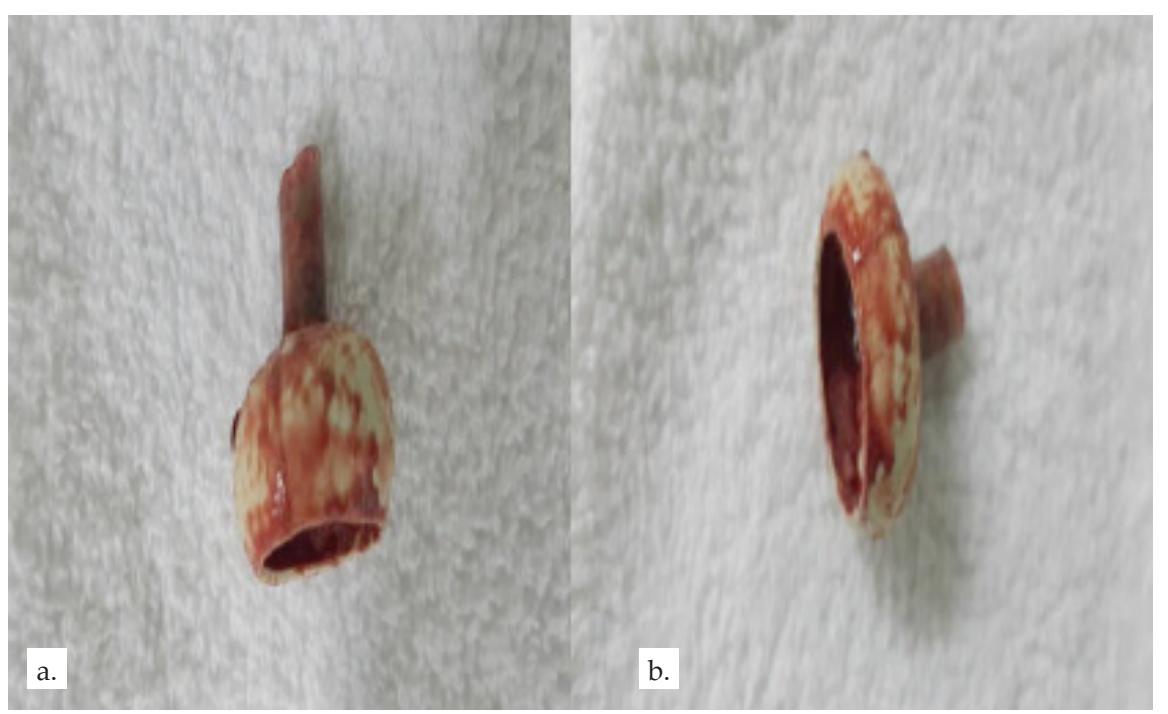


y hematoma gástricos, fistulización, neumonía por aspiración, absceso subcutáneo, síndrome del tope interno oculto, fascitis necrosante y pancreatitis aguda; entre las complicaciones leves, se incluyen infecciones de la herida, pérdida periestomal, hemorragia, neumoperitoneo, ulceración, obstrucción de la sonda y obstrucción de la salida gástrica. ${ }^{4-6,13}$ La infección de la herida es la complicación más frecuente cuando se descuidan la profilaxis antibiótica y la antisepsia. En una serie de Gauderer y col., hubo un $8 \%$ de complicaciones graves en un total de 224 procedimientos de GEP. ${ }^{5}$

Durante la GEP, se utilizan técnicas de tracción o empuje para la colocación, según lo que elija el personal médico, y no hay diferencias importantes entre ambas técnicas en cuanto a la aplicación y las tasas de complicaciones. ${ }^{11}$ Sin embargo, como se demostró en nuestro caso, con la técnica de "corte y empuje" la parte distal de la sonda de GEP puede desplazarse hasta el esófago en los pacientes con trastornos neurológicos, probablemente debido a la postura fija de los miembros, en lugar de que el tope de la sonda de GEP llegue hasta el estómago distal, como se espera en general que suceda. Independientemente del método utilizado, es necesario realizar un seguimiento regular del paciente, en especial para confirmar el pasaje correcto del tope de la sonda de GEP, y tomar imágenes radiológicas de la ubicación del tope, de ser necesario, para determinar si hay complicaciones y evitarlas. Las sondas de GEP deben ser antialérgicas y flexibles. Por lo tanto, las sondas de silicona o poliuretano son más aptas que las de polivinilo. ${ }^{11} \mathrm{Si}$ bien la sonda de GEP de nuestro paciente era de silicona, la utilización prolongada en el esófago causó la incrustación y el daño tisular. Debido a que la alimentación del paciente a través de una sonda de GEP que se ha reemplazado no compromete la alimentación enteral regular del paciente, la presencia de un cuerpo extraño en el esófago puede pasar desapercibida y puede no detectarse hasta que se produzca un daño tisular y hemorragia. Por lo tanto, deben realizarse estudios por imágenes durante el reemplazo de las sondas de GEP.
En este caso, después del reemplazo de la sonda de GEP mediante el método de corte y empuje, el tope se desplazó hasta el esófago, se incrustó en la mucosa y causó erosión, úlcera y hemorragia masiva. Si bien la GEP es un método seguro y fácil de implementar, es fundamental tomar imágenes endoscópicas o radiológicas después del procedimiento para reducir el riesgo de las complicaciones poco frecuentes que se presentaron en este caso.

\section{REFERENCIAS}

1. McWhirter JP, Pennington CR. Incidence and recognition of malnutrition in hospital. BMJ 1994;308(6934):945-8.

2. Heyland DK. Nutritional support in the critically ill patient: a critical review of the evidence. Crit Care Clin 1998;14(3):423-40.

3. Rahnemai-Azar AA, Rahnemaiazar AA, Naghshizadian R, y col. Percutaneous endoscopic gastrostomy: indications, technique, complications and management. World J Gastroenterol 2014;20(24):7739-51.

4. Gauderer M. Twenty years of percutaneous endoscopic gastrostomy: origin and evolution of a concept and its expanded applications. Gastrointest Endosc 1999;50(6): 879-82.

5. Gauderer MWL. Percutaneous endoscopic gastrostomy: a 10-year experience with 220 children. J Pediatr Surg 1991;26(3):288-94.

6. Schrag SP, Sharma R, Jaik NP, y col. Complications related to percutaneous endoscopic gastrostomy (PEG) tubes. A comprehensive clinical review. J Gastrointestin Liver Dis 2007;16(4):407-18.

7. Anagnostopoulos GK, Kostopoulos P, Arvanitidis DM. Buried bumper syndrome with a fatal out come, presenting early as gastrointestinal bleeding after percutaneous endoscopic gastrostomy placement. J Postgrad Med 2003;49(4):325-7.

8. Korula J, Harma C. A simple and inexpensive method of removal or replacement of gastrostomy tubes. JAMA 1991;265(11):1426-8.

9. Pearce CB, Goggin PM, Collett J, y col. The 'cut and push' method of percutaneous endoscopic gastrostomy tube removal. Clin Nutr 2000;19(2):133-5.

10. Peacock O, Singh R, Cole A, y col. The 'cut and push' technique: is it really safe? BMJ Case Rep 2012;2012:bcr2012006607.

11. Marik PE, Zaloga GP. Early enteral nutrition in acutely ill patients: asystematic review. Crit Care Med 2001;29(12):2264-70.

12. Ségal D, Michaud L, Guimber D, y col. Late onset complications of percutaneous endoscopic gastrostomy in children. J Pediatr Gastroenterol Nutr 2001;33(4):495-500.

13. Fortunato JE, Troy AL, Cuffari C, y col. Outcome after percutaneous endoscopic gastrostomy in children and young adults. J Pediatr Gastroenterol Nutr 2010;50(4):390-3. 